\title{
Propiedades Psicométricas y la Validación del Instrumento de Evaluación del Autoesquema Sexual Femenino en Mujeres Jóvenes Ecuatorianas
}

\author{
Psychometric Properties and Validation of the Women's Sexual Self-Schema \\ Evaluation in a Young Ecuadorian Sample
}

\author{
M. D. Brito-Rhor ${ }^{1}$, B. Rodríguez-Herráez ${ }^{2}$ y A. Trueba ${ }^{3}$
}

\begin{abstract}
Resumen
Se examinó cómo se autodescriben sexualmente las mujeres jóvenes ecuatorianas. La evaluación del autoesquema sexual femenino (ASF) permite entender la atracción o rechazo a la información o comportamiento sexual. Participaron 414 mujeres estudiantes de la Universidad San Francisco de Quito (Ecuador) con una edad promedio de 19.74 años, y todas completaron la escala ASF. El análisis de los resultados reveló que existen 3 factores que explicaron el $67.71 \%$ de la varianza total: erotismo, romanticismo y afectividad negativa. Las jóvenes se identificaron más con adjetivos vinculados al componente romántico de la sexualidad como: amorosa, cariñosa, cálida, tierna o romántica. Por otra parte, se identificaron menos con adjetivos de los componentes de afectividad negativa y erotismo como: reprimida, erótica, ardiente, cerrada y cohibida. La consistencia interna del instrumento para cada uno de los tres factores estimada a través del alfa de Cronbach fue: Erotismo $\alpha=.952$, Romanticismo $\alpha=.864$ y Afectividad Negativa $\alpha=.896$.
\end{abstract}

Palabras clave: autoconcepto sexual, autoesquema sexual femenino, personalidad, sexualidad femenina

\begin{abstract}
We examined how young Ecuadorian women describe themselves sexually. The evaluation of the female sexual self-schema (ASF) allowed us to understand the attraction or rejection to sexually-related information or behaviors. The participants were 414 women, students at Universidad San Francisco de Quito (Ecuador) with an average age of 19.74 years. They all completed the ASF scale. The results of our analysis indicated that there were 3 ASF factors that explained $67.71 \%$ of the total variance: eroticism, romanticism and negative affectivity. The young women tended to identify more with adjectives related to the romantic component of sexuality: loving, affectionate, warm, tender or romantic. On the other hand, they identify themselves less with adjectives related to negative affect and eroticism such as: repressed, erotic, ardent, closed and self-conscious. The internal consistency of the instrument for each of the three factors estimated through Cronbach's alpha was: Erotism $\alpha=.952$, Romanticism $\alpha=.864$ and Negative Affectivity $\alpha=.896$.
\end{abstract}

Keywords: female sexuality, personality, sexual self-concept, women's sexual self-schema

\footnotetext{
${ }^{1} \mathrm{PhD}$ en Ciencias Sociales y Jurídicas, MBA y BSBA. Professor, USFQ (Profesor Titular) y Doctor, URJC. Universidad Rey Juan Carlos (URJC), España.Universidad San Francisco de Quito (USFQ), Ecuador. Tel.: 59390302119. Correo: mbrito@usfq.edu.ec

2 Dra. Marketing. Professor (Profesora Contratada Doctor). Universidad Rey Juan Carlos, España. Tel.: 34616395764. Correo: beatriz.rodriguez@urjc.es

${ }^{3} \mathrm{PhD}$. en Psicología Clínica, MA en Psicología Clínica, BS en Neurociencia. Professor at USFQ. Universidad San Francisco de Quito, Ecuador. Tel.: +593999695378. Correo: atrueba@usfq.edu.ec
} 


\section{Introducción}

Las investigaciones en el campo de la sexología y la psicología social sugieren que los rasgos sexuales de la personalidad podrían predecir la atracción o rechazo hacia la información, la situación y el comportamiento sexual (Fisher, White, Byrne, \& Kelley, 1988; Reichert \& Fosu, 2005). Bajo estas premisas cabe suponer que la forma en que se definen las mujeres en el ámbito sexual dependerá de diferentes factores basados en los conocimientos previos y actuales de las experiencias que se hayan tenido en este aspecto, y que influirán en el procesamiento de información sexual y social relevante y guiarán el comportamiento sexual (Andersen \& Cyranowski, 1994).

El término autoesquema hace referencia a las creencias o ideas que una persona tiene de sí mismo (Mueller, Rehman, Fallis, \& Goodnight, 2015). De esta forma, se puede considerar que el autoesquema sexual femenino (ASF) es una variable que permite conocer la respuesta sexual y su correlación con las emociones en las relaciones de pareja, el modelo sexual, las actitudes y las creencias sexuales. Andersen y Cyranowski (1994) realizaron un estudio en la Universidad de Ohio en los Estados Unidos de América sobre la representación cognitiva que tienen las mujeres sobre la sexualidad. La cuestión principal de su investigación fue cómo se describen y consideran sexualmente las mujeres estadounidenses a sí mismas. Los resultados de su trabajo confirman que la percepción que tienen las mujeres sobre cuestiones sexuales proviene de experiencias pasadas. Estos autores pretendían construir y validar una escala de autoesquema sexual femenino que se formó a partir de tres factores relacionados con adjetivos tales como románticoapasionado, abierto-directo y avergonzadoconservador. Los dos primeros factores aparecían correlacionados entre sí y los consideraron como la dimensión positiva, mientras que el tercer factor correlacionó de manera inversa con los otros, por lo que se identificó como la dimensión negativa. La combinación de estas dos dimensiones proporciona un modelo bidimensional que permite clasificar a las mujeres en cuatro tipos: mujeres con autoesquema sexual positivo, mujeres con autoesquema sexual negativo, mujeres coesquemáticas (las que obtienen valores altos en las dos dimensiones) y mujeres aesquemáticas (las que obtienen valores bajos en las dos dimensiones).

Debido a la relevancia del tema del autoesquema sexual femenino y tomando como base el constructo propuesto por Andersen y Cyranowski (1994), las investigaciones han sido replicadas en diferentes países. Por ejemplo: en Australia (Wyllie, Carlson, \& Rosenberger, 2015), donde el objetivo de la réplica fue expandir el conocimiento contrastando la estructura factorial de la escala con los hallazgos originales de EE. UU.; y en China (Cheung, Chan, Han, Sze, \& Fan, 2013), donde se midieron los efectos de los rasgos de personalidad de las mujeres chinas ante la información sexual. Por otra parte, en México (Martínez Rodarte, Reyes Lagunes, \& Paredes Guerrero, 2010), se construyó y validó una escala del autoesquema sexual femenino que fuera culturalmente relevante y que permitiera evaluar la manera en que se autodescriben sexualmente las mujeres mexicanas. Precisamente, el presente estudio da continuidad a otras investigaciones realizados en otros países como México. Aunque México y el Ecuador comparten similitudes culturales son diferentes. Este estudio contribuye significativamente a entender las actitudes sexuales en un país en el que nunca se ha estudiado el autoesquema sexual. Es importante validarlo en el Ecuador porque el contexto cultural es distinto al mexicano y sabemos que los factores culturales alteran el autoesquema sexual (Rosen \& Beck, 1988).

En términos generales, los esquemas van a variar dependiendo de algunos factores como por ejemplo la cultura (Ramírez Esparza, Chung, Sierra Otero, \& Pennebaker, 2012) u otros elementos del entorno (Leite \& Kuiper, 2008). De acuerdo con García (1999), el autoesquema sexual es la descripción que la persona expresa sobre sus propios aspectos sexuales. Las percepciones cognitivas que tiene un ser humano sobre sí mismo en su sexualidad van a influir en las formas en las cuales un individuo procesa y responde en las situaciones sociales. Además, estas percepciones van a influir directamente en su conducta sexual (Andersen \& Cyranowski, 1994). El autoesquema sexual es una variable predictora, independiente y no manipulada, que 
sirve para entender otros estudios sobre la respuesta sexual, el rol de las emociones en las relaciones de pareja, el modelo sexual, las actitudes y creencias sexuales (Cyranowski \& Andersen, 1998). Estudiar el autoesquema femenino es importante porque no es igual que el masculino. Hay estudios que indican que en muchas culturas hay una supresión de la sexualidad femenina en comparación a la masculina (Baumeister \& Twenge, 2002). Es por eso importante estudiar el autoesquema femenino específicamente.

De acuerdo con la Organización Mundial de la Salud (OMS/WHO), se define la sexualidad como "un aspecto central del ser humano a lo largo de toda la vida, que abarca el sexo, las identidades y roles de género, el erotismo, el placer, la intimidad, la reproducción y la orientación sexual" (Molina \& Tejada, 2017, p.19). El componente de género, masculino o femenino, establece que los seres humanos están influenciados por su sexo biológico, hombre o mujer, y por el entorno en que se desarrollan (Orcasita, Cuenca, Montenegro, Garrido, \& Haderlein, 2018). La sexualidad es una variable presente en diversas culturas con sus respectivas diferencias. La sexualidad, la actitud y la autoevaluación son factores que van desde lo biológico hasta lo psicológico pasando por lo social. De esta forma, la actitud sexual se entiende como una reacción ante un cúmulo de experiencias culturales relacionadas directamente con la personalidad, razón por la cual ésta debe incluir variables que van más allá de los mecanismos fisiológicos y cognitivos (Rosen \& Beck, 1988).

A partir del estudio de Andersen y Cyranoski (1994) se definieron cuatro tipos de autoesquemas sexuales basados en la combinación de dos dimensiones independientes. Una primera dimensión relacionada con las emociones de pasión

Tabla 1. Tipos de autoesquemas sexuales femeninos de acuerdo con Andersen y Cyranowski (1994)

\begin{tabular}{lccc}
\hline & \multicolumn{2}{c}{ Dimensión Positiva } \\
\cline { 2 - 4 } & Baja & $\begin{array}{c}\text { Mujeres a- } \\
\text { esquemáticas. }\end{array}$ & $\begin{array}{c}\text { Mujeres con } \\
\text { autoesquema } \\
\text { positivo. }\end{array}$ \\
$\begin{array}{l}\text { Dimensión } \\
\text { Negativa }\end{array}$ & Alta & $\begin{array}{c}\text { Mujeres con } \\
\text { autoesquema } \\
\text { negativo. }\end{array}$ & $\begin{array}{c}\text { Mujeres co- } \\
\text { esquemáticas. }\end{array}$ \\
\hline
\end{tabular}

y romanticismo y la otra con el aspecto negativo de vergüenza o conservador. Este modelo bivariante da lugar a los cuatro autoesquemas mencionados que permiten identificar a las mujeres según su autoesquema (Tabla 1).

La percepción sexual que tienen las mujeres está relacionada con la cultura, dando escalas y factores diferentes según la nacionalidad de las entrevistadas. En el caso de las mujeres mexicanas su autoesquema está formado por cuatro factores:

1) erotismo; el componente sensual de la sexualidad. Este elemento se relaciona con las cogniciones congruentes de la capacidad de experimentar y provocar en el otro ser deseo y placer sexual.

2) romanticismo; el componente afectivoemocional de la sexualidad. Las cogniciones concernientes al romanticismo reflejan el afecto y la sensibilidad.

3) apertura sexual; el componente conductual de la sexualidad. En la conducta de la persona, las cogniciones son vinculadas con la seguridad, apertura, asertividad y confianza en la competencia sexual personal.

4) afectividad negativa; el componente afectivo-emocional y conductual negativo de la sexualidad. En este elemento las cogniciones congruentes están relacionados con sentimientos de tensión, ansiedad y culpa estimulados por aspectos sexuales que podrían ocasionar comportamientos de contención sexual (Martínez Rodarte et al., 2010).

Existen estudios que indican que las mujeres con autoesquema sexual positivo poseen características como la entrega, el entusiasmo y el romanticismo, además de mostrar una actitud sexual más liberal y el aumento de su interés en cuestiones sexuales (Andersen \& Cyranowski, 1994; Cheung et al., 2013; Martínez Rodarte et al., 2010; Wyllie et al., 2015). Este grupo de mujeres demuestra más libertad en las restricciones sociales como, por ejemplo, ellas no se avergüenzan al hablar de temas sexuales. A pesar de la profundidad de las investigaciones realizadas, sus resultados no se pueden generalizar por haber empleado parámetros diferentes en cada estudio.

Con el objetivo de describir el autoconcepto sexual de las jóvenes y comprobar la validez de los resultados de los trabajos anteriores en el 
entorno ecuatoriano, se decidió realizar el estudio utilizando los mismos instrumentos de la investigación. La decisión de adaptación de la escala mexicana de medición (Martínez Rodarte et al., 2010), responde al hecho de que la población mexicana es culturalmente más cercana y relevante en sus comportamientos a la ecuatoriana. La proximidad entre los dos entornos investigados permite suponer que en el caso de las mujeres ecuatorianas se obtendrán los mismos o por lo menos parecidos comportamientos referidos al ASF.

La presente investigación tiene como objetivo hacer una evaluación psicométrica y validación del constructo ASF de las jóvenes ecuatorianas. Para poder hacerlo se han definido los autoesquemas sexuales de acuerdo con las cuatro tipologías expuestas por Andersen y Cyranowski (1994) en la evaluación del autoesquema sexual femenino.

Este trabajo consiste en identificar como se autodescriben y consideran sexualmente las mujeres ecuatorianas. Dada la cercanía cultural entre México y Ecuador se decidió utilizar el mismo instrumento que Martínez Rodarte et al. (2010) aunque se introdujo alguna adaptación propia en base a los resultados de las encuestas.

\section{Método}

\section{Participantes}

La técnica de muestreo utilizada fue la de conveniencia no probabilística. La muestra estuvo formada por 414 mujeres, de nacionalidad ecuatoriana, estudiantes de pregrado de diversas facultades de una universidad privada en el Ecuador (Universidad San Francisco de Quito - USFQ), con edades comprendidas entre 17 y 27 años $(M=19.74$, $\mathrm{SD}=1.89$ ). La tasa de respuesta fue del $73 \%$. Los criterios de inclusión fueron tanto la capacidad para leer y escribir como la de entender la encuesta. Se consideraron como criterios de exclusión el no tener la nacionalidad ecuatoriana y ser mayor de 27 años. Se realizó el estudio en octubre del 2016. En la Tabla 2 se exhiben las características sociodemográficas relevantes de la muestra.
Tabla 2. Características sociodemográficas de la

\begin{tabular}{lc}
\multicolumn{2}{c}{ muestra } \\
\hline Características & Descripción \\
\hline Edad & $19.74(1.89)$ \\
Sedia (DT) & Femenino \\
Nacionalidad & Ecuatorianas \\
Estado Civil & Solteras (98.73\%) \\
Nivel de estudios & Cursando Universidad \\
& Ingenierías (19.06\%), Psicología \\
Carreras & $(15.3 \%)$, Medicina (8.9\%), \\
& Administración de Empresas (6.28\%), \\
& Economía (5.37\%), otros (45.09\%).
\end{tabular}

\section{Instrumentos}

La herramienta elegida para recolectar los datos ha sido un cuestionario de auto-reporte. Se utilizaron dos instrumentos: uno sociodemográfico elaborado ad hoc y el otro que evalúa el autoesquema sexual femenino (ASF).

Cuestionario Sociodemográfico. Se recogen datos de clasificación de carácter sociocultural para identificar a los integrantes de la muestra. Incluye preguntas sobre sobre sexo, edad, estado civil, nacionalidad y carrera que cursa.

Versión mexicana del Autoesquema Sexual Femenino (ASF). Inicialmente, el instrumento fue desarrollado por Andersen y Cyranowski (1994), escala que fue construida y validada en población estadounidense. No obstante, para facilitar y garantizar un adecuado grado de entendimiento y compresión de las preguntas, se utilizó la versión en español, construida y validada para población mexicana por Martínez Rodarte et al. (2010). La propuesta definitiva del cuestionario fue evaluada, revisada y modificada por profesores ecuatorianos expertos en lenguaje y psicología de la USFQ. Se solicitó a los investigadores académicos que proporcionaran comentarios sobre la redacción y la validez de los ítems. En la versión final del cuestionario se incorporaron sugerencias recibidas para aumentar la claridad y la exhaustividad de las preguntas. Se incluye las preguntas relacionadas directamente con los rasgos sexuales de la personalidad determinados a través de varios adjetivos representativos del erotismo, el romanticismo, la apertura sexual y la afectividad negativa pertinentes al autoesquema sexual femenino y adoptados de la escala mexicana. Cada persona 
realizó su auto-descripción en base a 32 adjetivos evaluados a través de una escala de Likert de 7 puntos, donde 0 es que ese adjetivo es "Nada descriptivo" y 7 que es "Muy descriptivo" (Apéndice 1). El rango del índice del autoesquema sexual en la escala aplicada podría ser máximo de 101 y mínimo de -61. Es decir, que el punto medio o mediana estaría en 20.

La realización del cuestionario ha sido aplicada en forma de encuestas personales. La aplicación ad personam del cuestionario, ha permitido un aumento del nivel de control del proceso de las respuestas, evitando las influencias externas de otras personas y del entorno en general (Babbie, 2000).

\section{Procedimiento}

Se contactó previamente a un docente representante de cada una de las 48 carreras universitarias ofrecidas en la USFQ con el fin de conseguir un relativo equilibrio en los sujetos encuestados. El docente debía aceptar que sus alumnos fueran encuestados y el estudiante también debía consentir explícitamente su participación voluntaria en el estudio. Se programó la recolección de datos para el mes de octubre del 2016 con el objetico de evitar el período de exámenes que es una época de alto estrés para los estudiantes. El encuestador se presentaba en el aula y daba las instrucciones personalmente. Los sujetos recibían un hipervínculo, cargado en una plataforma de aprendizaje llamada D2L, que los redirigía a contestar el cuestionario directamente en Qualtrics, software que facilita el manejo de encuestas. Las alumnas utilizaron dispositivos móviles (celulares inteligentes o tabletas) para completar las preguntas. Las instrucciones dadas fueron muy precisas y no hacían referencia directa a la autodescripción sexual para evitar sesgo en la respuesta. Las indicaciones fueron las siguientes: "Descríbete a ti misma. Debajo está una lista de 32 adjetivos. Para cada palabra, considera si el término te describe o no. Cada adjetivo se puede calificar en una escala que va de $1=$ Nada descriptivo a 7= Muy descriptivo. Elije un número para cada adjetivo para indicar que tan preciso éste te describe. Pregunta: ¿En qué medida me describe el término ?".
Las 32 variables para definir el autoesquema sexual son las siguientes: caliente, ardiente, seductora, excitable, sexy, sensual, provocativa, excitante, erótica, coqueta, deseosa, tierna, cariñosa, romántica, amorosa, cálida, creativa, divertida, juguetona, imaginativa, temerosa, nerviosa, angustiada, insegura, tensa, tímida, penosa, cohibida, reprimida, recatada, inhibida y cerrada.

\section{Análisis de datos}

Análisis de validez y consistencia interna Con el objetivo de efectuar el estudio de validez, se realizó un análisis factorial exploratorio de los ítems (AFE). Utilizando los nuevos avances psicométricos recomendados por Lloret Segura, Ferreres Traver, Hernández Baeza y Tomás Marco (2014), se sometieron los 32 ítems a un análisis factorial con el procedimiento Análisis Paralelo para determinar el número de dimensiones, con el método de extracción de mínimos cuadrados no ponderados y con rotación oblicua Oblimin Ponderado (Lorenzo Seva, 2000) para alcanzar la simplicidad de factores. Con esto se pretende determinar la validez del instrumento. Adicionalmente, a través del alfa de Cronbach se obtuvo la consistencia interna del instrumento. Los softwares utilizados fueron SPSS, STATA y FACTOR que son programas desarrollados para ajustarse al modelo de Análisis Exploratorio de Factores.

\section{Resultados}

La matriz de componentes pasó de tener 32 ítems a 27, los mismos que quedaron agrupados en alguno de los tres factores. Se examinó la validez de los 27 elementos utilizados para medir el autoesquema sexual y se obtuvo un KMO de .923. Al ser KMO mayor que .60 se pudo concluir que existe una correlación de cada par de reactivos con el resto de los elementos. Al realizar la prueba de esfericidad de Bartlett se obtuvo una significancia de $p=.001$, es decir, se confirma que existe una correlación significativa y el análisis factorial es pertinente.

A continuación, se efectuó un análisis para determinar los factores de este instrumento y las 
Tabla 3. Matriz de componentes rotados* del autoesquema sexual femenino

\begin{tabular}{|c|c|c|c|}
\hline & $\begin{array}{c}1 \\
\text { Erotismo }\end{array}$ & $\begin{array}{c}2 \\
\text { Romanticismo }\end{array}$ & $\begin{array}{c}3 \\
\text { Afectividad } \\
\text { Negativa }\end{array}$ \\
\hline Caliente & .740 & .04 & .109 \\
\hline Ardiente & .758 & .02 & .102 \\
\hline Seductora & .800 & -.086 & .147 \\
\hline Excitable & 699 & .075 & .081 \\
\hline Sexy & .730 & -.121 & .188 \\
\hline Sensual & .797 & -.088 & .139 \\
\hline Provocativa & .784 & -.037 & .102 \\
\hline Excitante & .760 & -.020 & .093 \\
\hline Erótica & .749 & .048 & .029 \\
\hline Coqueta & .570 &,- 124 & .262 \\
\hline Deseosa & .541 & .061 & .190 \\
\hline Tierna & .111 & .663 & .092 \\
\hline Cariñosa & .112 & .868 & .041 \\
\hline Romántica & .213 & .718 & .099 \\
\hline Amorosa & .162 & .891 & .006 \\
\hline Cálida & .272 & .647 & .061 \\
\hline Temerosa & .041 & .098 & .723 \\
\hline Nerviosa & -.049 & .138 & .736 \\
\hline Angustiada & -.009 & .027 & .661 \\
\hline Insegura & -.064 & -.029 & .709 \\
\hline Tensa & -.017 & .017 & .585 \\
\hline Tímida & -.207 & .096 & .693 \\
\hline Vergonzosa & -.091 & .084 & .658 \\
\hline Cohibida & .002 & .052 & .723 \\
\hline Reprimida & .087 & .033 & .690 \\
\hline Inhibida & .213 & -.013 & .578 \\
\hline Cerrada & -.054 & -.057 & .524 \\
\hline
\end{tabular}

Nota. *Las cargas factoriales mayores 0.50 están en negrita. Procedimiento para determinar el número de dimensiones: Implementación óptima del Análisis Paralelo (PA) (Timmerman \& Lorenzo Seva, 2011). Método para la extracción de factores: Cuadrados mínimos no ponderados (ULS). Rotación para lograr la simplicidad factorial: Oblimina ponderada (Lorenzo Seva, 2000). Se eliminaron 5 ítems del ASF para mujeres mexicanas: Creativa, Divertida, Juguetona, Imaginativa y Recatada.

cargas factoriales de los adjetivos. En la matriz de componentes rotados expuesta se puede apreciar que hay tres factores o componentes (Tabla 3). Tomando el criterio de Hair, Anderson, Tatham y Black (1999), solo se tuvieron en cuenta los elementos con cargas factoriales iguales $\mathrm{o}$ mayores que .50 mientras que aquellos que mostraban cargas por debajo de 0.50 fueron eliminados.

Una vez obtenidos los factores, se los rota para poder tener una interpretación más clara de los mismos. El método de rotación utilizado en este trabajo ha sido oblicuo, específicamente el Oblimin Ponderado (Lorenzo Seva, 2000). Se encontró que tres factores fueron los que explicaron el autoesquema sexual femenino de las mujeres ecuatorianas. Es decir, que los reactivos del instrumento deben quedar agrupados en alguno de esos tres factores. De acuerdo con varias investigaciones (Fabrigar, Wegener, MacCallum, \& Strahan, 1999; Ferrando \& Anguiano Carrasco, 2010) se establece que debe existir un mínimo de 3 o 4 ítems por factor. Por lo tanto, con el objetivo de tener una solución factorial sólida, se decidió eliminar cualquier factor que no cumpliera con estas condiciones.

El autoesquema sexual para mujeres jóvenes ecuatorianas estaría compuesto por 3 factores con 27 ítems como se describe a continuación:

Factor1 (Erotismo): caliente, ardiente, seductora, excitable, sexy, sensual, provocativa, excitante, erótica, coqueta, deseosa.

Factor2 (Romanticismo): tierna, cariñosa, romántica, amorosa, cálida.

Factor3 (Afectividad Negativa): temerosa, nerviosa, angustiada, insegura, tensa, tímida, penosa, cohibida, reprimida, inhibida, cerrada.

Se obtuvo un alfa de Cronbach aceptable para cada uno de los tres factores que la conforman: Erotismo $\alpha=.952, \quad$ Romanticismo $\alpha=.864 \quad \mathrm{y}$ Afectividad Negativa $\alpha=.896$ ) (Tabla 4).

Tabla 4. Autoesquema sexual femenino de las jóvenes ecuatorianas

\begin{tabular}{lcccccc}
\hline Factor & Ítems & $\begin{array}{c}\text { Factor } \\
(\alpha)\end{array}$ & $M$ & DT & Min & Max \\
\hline 1. Erotismo & 11 & .952 & 37.07 & 15.45 & 11 & 77 \\
2. Romanticismo & 5 & .864 & 25.01 & 6.60 & 5 & 35 \\
3. Afectividad & 11 & .896 & 37.50 & 12.92 & 11 & 77 \\
negativa & 27 & .888 & 24.58 & 22.73 & -46 & 93 \\
ASF &
\end{tabular}

Nota. ASF=Índice Autoesquema Sexual (Factor1+Factor2Factor3); $=414$

Tabla 5. Correlaciones entre los factores de la Escala de Autoesquema sexual femenino

\begin{tabular}{lccc}
\hline Factor & Erotismo & Romanticismo & $\begin{array}{c}\text { Afectividad } \\
\text { negativa }\end{array}$ \\
\hline 1. Erotismo & 1 & & \\
2. Romanticismo & $.351^{* *}$ & 1 & \\
3. Afectividad & -.040 & $.117^{*}$ & 1 \\
negativa & $.804 * *$ & $.462 * *$ & $-.561^{* *}$ \\
ASF &
\end{tabular}

Nota. **La correlación es significativa al nivel .01 (bilateral) *La correlación es significativa al nivel .05 (bilateral). ASF= Índice Autoesquema Sexual

De acuerdo con el modelo desarrollado por Andersen y Cyranowski (1994) y tomando en cuenta las correlaciones calculadas en este estudio (Tabla 5) para obtener un índice de la variable autoesquema sexual se sumaron las calificaciones del Factor 1: erotismo y del Factor 2: romanticismo y se restaron las del Factor 3: 
afectividad negativa. En el siguiente paso se calculó el promedio del índice de autoesquema sexual que resultó de 24.58 con una desviación estándar de 22.73 (Tabla 4). El índice promedio del estudio $(M=24.58)$ del ASF de las ecuatorianas está sobre la mediana de 20.

Tabla 6. Promedio de los adjetivos del autoesquema sexual de jóvenes ecuatorianas

\begin{tabular}{lcc}
\hline Item & Factor & Media (s) \\
\hline 15. Amorosa & Romanticismo & $5.20(1.609)$ \\
13. Cariñosa & Romanticismo & $5.17(1.593)$ \\
16. Cálida & Romanticismo & $4.95(1.553)$ \\
12. Tierna & Romanticismo & $4.91(1.653)$ \\
14. Romántica & Romanticismo & $4.78(1.793)$ \\
18. Nerviosa & Afectividad negativa & $4.05(1.713)$ \\
10. Coqueta & Erotismo & $4.03(1.726)$ \\
21.Tensa & Afectividad negativa & $3.82(1.706)$ \\
22. Tímida & Afectividad negativa & $3.80(1.854)$ \\
23. Vergonzosa & Afectividad negativa & $3.72(1.720)$ \\
11.Deseosa & Erotismo & $3.68(1.780)$ \\
5. Sexy & Erotismo & $3.64(1.692)$ \\
19. Angustiada & Afectividad negativa & $3.57(1.668)$ \\
20. Insegura & Afectividad negativa & $3.54(1.765)$ \\
17. Temerosa & Afectividad negativa & $3.48(1.627)$ \\
6. Sensual & Erotismo & $3.47(1.707)$ \\
4. Excitable & Erotismo & $3.42(1.772)$ \\
3. Seductora & Erotismo & $3.34(1.698)$ \\
8. Excitante & Erotismo & $3.34(1.692)$ \\
7. Provocativa & Erotismo & $3.25(1.679)$ \\
1. Caliente & Erotismo & $3.07(1.701)$ \\
26. Inhibida & Afectividad negativa & $3.05(1.587)$ \\
24. Cohibida & Afectividad negativa & $3.01(1.568)$ \\
27. Cerrada & Afectividad negativa & $2.99(1.724)$ \\
2. Ardiente & Erotismo & $2.95(1.647)$ \\
9. Erótica & Erotismo & $2.88(1.703)$ \\
25. Reprimida & Afectividad negativa & $2.48(1.474)$ \\
\hline & &
\end{tabular}

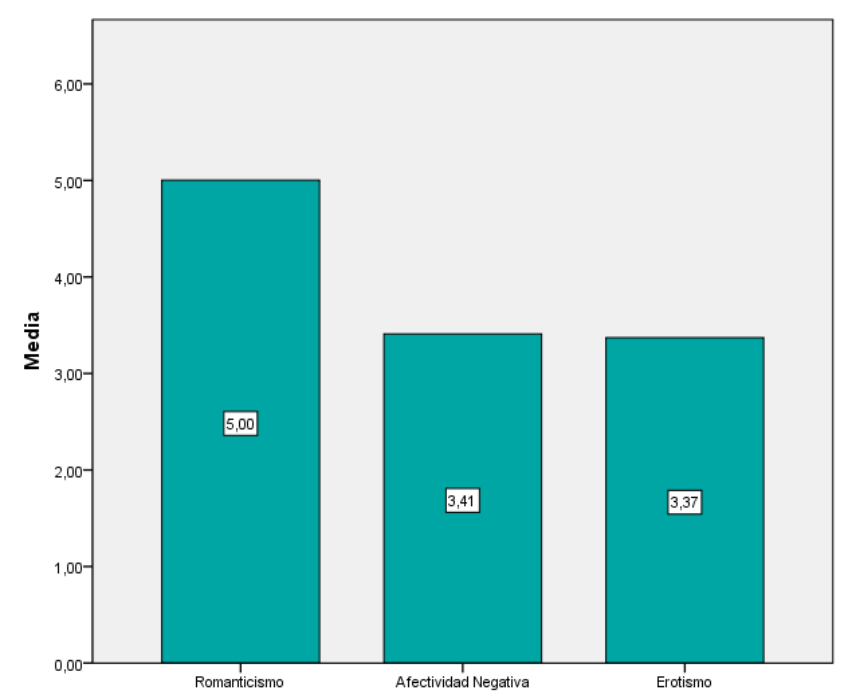

Figura 1. Autoesquema sexual femenino de jóvenes ecuatorianas

Se pudo concluir que la media del Factor romanticismo $(M=5.00)$ es significativamente diferente comparada con la media del Factor erotismo $(M=3.37, p<.001)$ y con la media del Factor afectividad negativa $(M=3.40, p<.001)$. Sin embargo, no se consiguió concluir que la media del Factor erotismo sea diferente al de la afectividad negativa $(p<.672)$. Los resultados indican que las mujeres jóvenes ecuatorianas se definen sexualmente a través de adjetivos de romanticismo como amorosa, cariñosa, cálida, tierna y romántica. En contraste, se identifican menos con adjetivos de erotismo y afectividad negativa como reprimida, erótica, ardiente, cerrada y cohibida (Figura 1, Tabla 6).

La clasificación que se puede hacer de las mujeres según los valores obtenidos, y siempre bajo el modelo bidimensional propuesto por Andersen y Cyranowsky (1994), muestra que el $33.8 \%$ de las mujeres son aesquemáticas, es decir que tanto los factores positivos como negativos están por debajo de la media. El 31.6\% manifiesta un autoesquema sexual positivo, es decir que los factores positivos están sobre la media y los negativos están por debajo de la media. Adicionalmente, un $17.90 \%$ de las mujeres tiene un autoesquema sexual negativo (dimensión positiva baja y dimensión negativa alta) y un $16.70 \%$ son coesquemáticas (dimensión positiva alta y dimensión negativa alta) (Figura 2).

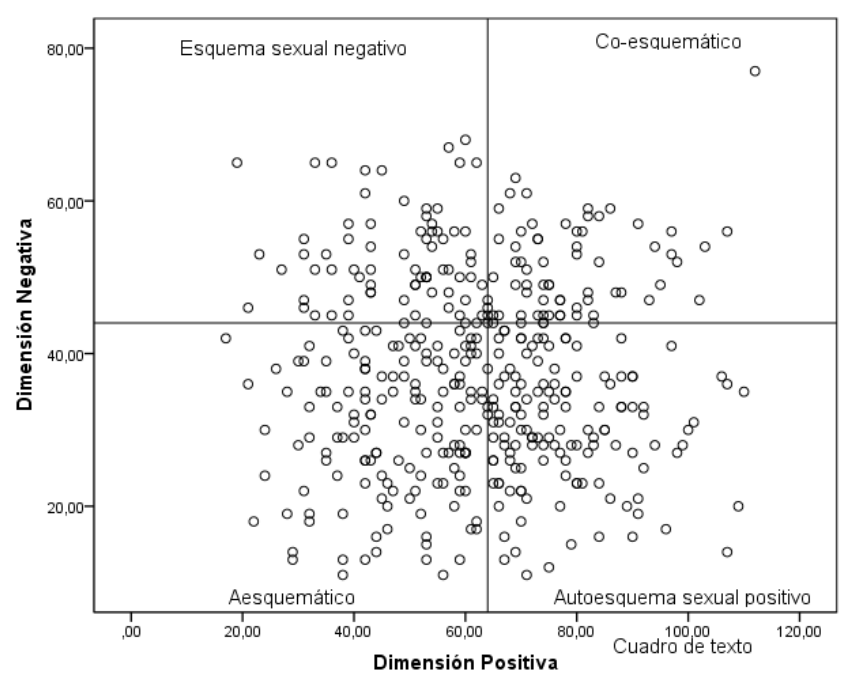

Figura 2. Dimensión positiva y negativa del índice del autoesquema sexual

\section{Discusión}

Esta investigación analiza las propiedades psicométricas y la validación del instrumento de evaluación del autoesquema sexual femenino de 
las jóvenes ecuatorianas. Se reveló cómo ellas se autodescriben y consideran sexualmente. La literatura en el campo de la sexología y la psicología social sugiere que el autoesquema sexual de las jóvenes ecuatorianas podría predecir su atracción o rechazo hacia la información, la situación y el comportamiento sexual. Dada la cercanía cultural entre México y Ecuador se decidió utilizar el mismo instrumento que Martínez Rodarte et al. (2010) aunque se introdujo alguna adaptación propia en base a los resultados de las encuestas.

Los resultados de la investigación realizada permiten considerar que el autoesquema sexual de las jóvenes ecuatorianas se caracteriza como cognitivo con aspectos positivos y negativos. El romanticismo, el erotismo y la afectividad negativa conforman los tres factores culturalmente relevantes que miden el autoesquema sexual de las jóvenes ecuatorianas. Con un Alfa de Cronbach mayor que .70 en todas las escalas se muestra una fiabilidad por consistencia interna aceptable para comparar grupos (Nunnally \& Bernstein, 1994).

Resumiendo, el estudio realizado ha mostrado que el modelo de autoesquema sexual de las mujeres jóvenes ecuatorianas incluye tres componentes: (1) erótico o pasional, (2) romántico y (3) afectivo negativo o conservador. Bajo un modelo bidimensional, se determinó que existen dos componentes positivos ( 1 y 2 ) y uno negativo (3) que conformarían el índice del autoesquema sexual femenino para jóvenes ecuatorianas.

El factor romanticismo ocupa el primer lugar como factor relevante en la descripción que hacen las mujeres ecuatorianas de sus rasgos sexuales de personalidad. Nuestro estudio expone que los principales adjetivos que lo conforman están relacionados con comportamientos cariñosos y amorosos. Los resultados de la investigación realizada en México indican como los adjetivos más consistentes de este factor el afecto y la sensibilidad (Martínez Rodarte et al., 2010). Las mujeres ecuatorianas le ven al factor romántico como lo más representativo en cuanto a su sexualidad esto puede ser el producto del ámbito cultural y estereotipos de género. Un metaanálisis realizado en EEUU sustenta esta postura, mostrando que las mujeres están más enfocadas en la parte emocional de la sexualidad y los hombres más en la gratificación física (Leigh, 1989). Esto es común en culturas dominadas por el patriarcado y el androcentrismo.

El factor erotismo para población ecuatoriana, conformado por las cogniciones relacionadas con la proactividad y sensualidad ha sido el siguiente factor positivo relevante en la descripción que hacen las mujeres ecuatorianas. En el estudio exploratorio, los adjetivos que lo conformaron siguieron a los anteriores (Andersen \& Cyranowski, 1994), en orden de aparición y frecuencia de mención. En la escala homóloga mexicana, en este factor las cogniciones prevalecientes han sido relacionadas con la capacidad de experimentar y despertar en el otro deseo y placer sexual (Martínez Rodarte et al., 2010).

El tercer factor que representa los aspectos negativos del autoesquema sexual de las jóvenes ecuatorianas está conformado por los comportamientos cognitivos como temerosa y nerviosa. En la escala mexicana este factor denominado afectividad negativa representa las cogniciones relacionadas con los comportamientos como vergüenza, culpabilidad y tensión, así como actitudes conservadoras y conductas inhibidas (Martínez Rodarte et al., 2010). Los resultados de los factores de erotismo y afectividad negativa indican que las mujeres ecuatorianas tienen una dialéctica. Por un lado indican que tienen deseo sexual y a la vez también tienen afecto negativo hacia la sexualidad, es decir sentimientos de tensión, ansiedad y culpa. Aun así es importante resaltar que los aspectos negativos del autoesquema sexual están por debajo de la media. Esto indica que las mujeres ecuatorianas universitarias no se influencian tanto por el entorno cultural patriarcal que fomenta estas actitudes negativas hacia la sexualidad. Como hemos indicado anteriormente estudios indican que en general hay una supresión de la sexualidad femenina en comparación a la masculina (Baumeister \& Twenge, 2002).

Si comparamos los resultados del estudio realizado en México al ecuatoriano, podemos observar que los factores relacionados con romanticismo y erotismo muestran una influencia muy semejante en las dos poblaciones investigadas. El autoesquema sexual de las 
mujeres ecuatorianas y mexicanas en su vertiente positiva es muy parecido. En el caso del tercer factor relacionado con los autoesquemas negativos, se puede observar una diferencia importante e interesante. Suponemos que las experiencias, las influencias culturales y la personalidad en ambas poblaciones pueden producir estas diferencias en el concepto sexual de las mujeres. Las diferencias observadas confirman por un lado la importancia de considerar aspectos culturales en la medición del autoesquema sexual femenino sugeridas por Reyes y García (2008).

Por otro lado, nuestros resultados sugieren también que, en el momento de definir la sexualidad, no basta únicamente con el estudio de componentes fisiológicos y/o cognitivos, sino que también es necesaria la investigación de la variable autoconcepto sexual. El conocimiento del autoesquema sexual de las jóvenes ecuatorianas va a permitir entender o predecir de mejor manera la atracción o rechazo a la información, situación o comportamiento sexual. La diferencia en autoesquema sexual entre la población ecuatoriana y mexicana indica la importancia de realizar este tipo de estudio en diferentes poblaciones latinas ya que cada contexto cultural es distinto.

\section{Limitaciones y futuras investigaciones}

Nuestra investigación es la primera exploración sobre los rasgos sexuales de la personalidad o autoesquema sexual de las mujeres ecuatorianas. Por ser primera tiene una población de estudio restringida, lo que se convierte en su limitación. La procedencia de las mujeres encuestadas - el ámbito universitario, restringe también la generalización de los resultados. Asimismo, hay que tomar en cuenta que el método de muestreo no probabilístico no brinda a todos los sujetos de la población iguales oportunidades de ser elegidos.

Debido a la falta de estudios existentes en Ecuador sobre esta temática, los resultados de este estudio, como en muchas otras investigaciones, deben ser considerados con cautela ya que no es posible contrastar los resultados obtenidos con otras investigaciones en este contexto cultural específico. A pesar de ello, esto no invalida la importancia de esta investigación como punto de partida para nuevos estudios.

Una limitación de esta investigación es que se ha reducido la prueba original, eliminando algunos ítems, por lo que los resultados aquí informados parten del supuesto de que las escalas recortadas mantienen las mismas propiedades que tenían en la escala original. No obstante, serán precisos más estudios para mostrar que esto es así.

En futuros estudios sería muy interesante ampliar la muestra e incluir poblaciones ecuatorianas de diferentes edades, razas, nivel de educación, ubicación geográfica, nivel socioeconómico, que podrían arrojar unos resultados de carácter general. Asimismo, sería de mucha utilidad probar el presente instrumento en diferentes poblaciones latinoamericanas con el objetivo de evaluar tanto la consistencia interna como la validez del instrumento presentado en esta investigación a nivel regional. Adicionalmente, sería importante establecer en una propuesta de diseño longitudinal, si las representaciones sobre los aspectos sexuales de las mujeres ecuatorianas se mantienen en el tiempo o se deben a las incidencias del ciclo vital.

Creemos que el conocimiento de la correlación y la influencia de los rasgos de personalidad de las mujeres, ayudarían a reducir los efectos negativos de los comportamientos presentes en la sociedad ecuatoriana. Este conocimiento aumentaría las oportunidades para desarrollar una base de análisis y definición del modelo social de igualdad. Por lo tanto, creemos que sería muy oportuno la realización de más investigaciones centradas en los efectos específicos de autoesquema sexual femenino en varios aspectos de la vida social.

\section{Referencias}

Andersen, B. L., \& Cyranowski, J. M. (1994). Women's sexual self-schema. Journal of Personality and Social Psychology, 67(6), 1079-1100. doi:https://doi.org/10.1037/0022-3514.67.6. 1079

Babbie, E. (2000). Fundamentos de la investigación social. México: International. Thompson. 
Baumeister, R. F., \& Twenge, J. M. (2002). Cultural suppression of female sexuality. Review of General Psychology, 6(2), 166-203.

Cheung, M., Chan, A. S., Han, Y. M., Sze, S. L., \& Fan, N. H. (2013). Differential effects of Chinese women's sexual self-schema on responses to sex appeal in advertising. Journal of Promotion Management, 19(3), 373-391. doi:https://doi.org/10.1080/10496491.2013.78 7382

Cyranowski, J. M., \& Andersen, B. L. (1998). Schemas, sexuality, and romantic attachment. Journal of Personality and Social Psychology, 74(5), 1364-1379. doi:http://dx.doi.org/10.1037/00223514.74.5.1364

Cyranowski, J. M., \& Andersen, B. L. (2000). Evidence of self-schematic cognitive processing in women with differing sexual self-views. Journal of Social and Clinical Psychology, 19(4), 519-543.

doi:http://dx.doi.org.ezbiblio.usfq.edu.ec/10.1 521/jscp.2000.19.4.519

Fabrigar, L. R., Wegener, D. T., MacCallum, R. C., \& Strahan, E. J. (1999). Evaluating the use of exploratory factor analysis in psychological research. Psychological Methods, 4(3), 272299.

Ferrando, P. J., \& Anguiano Carrasco, C. (2010). El análisis factorial como técnica de investigación en psicología. Papeles del Psicólogo, 31(1), 18-33.

Fisher, W., White, L., Byrne, D., \& Kelley, K. (1988). Erotophobia-Erotophilia as a Dimension of Personality. The Journal of Sex Research, 25(1), 123-151. doi:http://dx.doi.org/10.1080/0022449880955 1448

García, L. T. (1999). The certainty of the sexual self-concept. The Canadian Journal of Human Sexuality, 8(4), 263.

Hair, J. F., Anderson, R. E., Tatham, R. L., \& Black, W. C. (1999). Análisis Multivariante (Quinta ed.). Madrid, España: Prentice Hall.

Hu, L.-T., \& Bentler P. M. (1999). Cut-off criteria for fit indexes in covariance structure analysis: Conventional criteria versus new alternatives, Structural Equation Modeling: A Multidisciplinary Journal, 6(1), 1-55. doi:https://doi.org/10.1080/107055199095401 18

Leigh, B. C. (1989). Reasons for having and avoiding sex: Gender, sexual orientation, and relationship to sexual behavior. Journal of Sex Research, 26(2), 199-209. doi:http://dx.doi.org/10.1080/0022449890955 1506

Leite, C., \& Kuiper, N. A. (2008). Positive and negative self-worth beliefs and evaluative standards. Europe's Journal of Psychology, 4(2). doi: https://doi.org/10.5964/ejop.v4i2.428

Lloret Segura, S., Ferreres Traver, A., Hernández Baeza, A., \& Tomás Marco, I. (2014). El análisis factorial exploratorio de los ítems: Una guía práctica, revisada y actualizada. Anales de Psicología, 30(3), 1151-1169. doi:https://dx.doi.org/10.6018/analesps.30.3.1 99361

Lorenzo Seva, U. (2000). The weighted oblimin rotation. Psychometrika, 65, 301-318.

Martínez Rodarte, I., Reyes Lagunes, I., \& Paredes Guerrero, R. (2010). Autoesquema sexual femenino: Construcción y validación de una escala para población Mexicana. Revista Iberoamericana de Diagnóstico y Evaluación - e Avaliação Psicológica, 2 (30), 143-155.

Molina, A. B., \& Tejada, A. J. R. (2017). Instrumentos de medida de actitudes hacia la sexualidad: Una revisión bibliográfica sistemática. Revista Iberoamericana de Diagnóstico y Evaluación - e Avaliação Psicológica, 1(43), 17-32. doi:https://doi.org/10.21865/RIDEP43_17

Mueller, K., Rehman, U. S., Fallis, E. E., \& Goodnight, J. A. (2015). An interpersonal investigation of sexual self-schemas. Archives of Sexual Behavior, 45 (2), 281-290. doi:https://doi.org/10.1007/s10508-015-0638-6

Nunnally, J. C., \& Bernstein, I. H. (1994). Psychological theory (3rd ed.), McGraw-Hill, New York.

Orcasita, L. T., Cuenca, J., Montenegro, J. L., Garrido, D., \& Haderlein, A. (2018). Diálogos y saberes sobre sexualidad de padres con hijos 
e hijas adolescentes escolarizados. Revista Colombiana de Psicología, 27, 41-53. doi:https://doi.org/10.15446/rcp.v27n1.62148

Ramírez Esparza, N., Chung, C. K., Sierra Otero, G., \& Pennebaker, J. W. (2012). Crosscultural constructions of self-schemas: Americans and Mexicans. Journal of CrossCultural Psychology, 43(2), 233-250.

Reichert, T., \& Fosu, I. (2005). Women's responses to sex in advertising: Examining the effect of women's sexual self-schema on responses to sexual content in commercials. Journal of Promotion Management, 11 (2-3), 143-153. doi:http://dx.doi.org/10.1300/J057v11n02_10

Reyes Lagunes, I., \& García, L. F. (2008). Procedimiento de validación psicométrica culturalmente relevante: Un ejemplo. La Psicología Social en México, 12, 625-630.

Rosen, R. C., \& Beck, J. G. (1988). Patterns of sexual arousal: Psychophysiological processes and clinical applications. New York: Guilford Press.

Timmerman, M. E., \& Lorenzo-Seva, U. (2011). Dimensionality assessment of ordered polytomous items with parallel analysis. Psychological Methods, 16(2), 209-20. doi:10.1037/a0023353

Wiederman, M. W., \& Hurst, S. R. (1997). Physical attractiveness, body image, and women's sexual self- schema. Psychology of Women Quarterly, 21(4), 567-580.

doi:http://dx.doi.org/10.1111/j.14716402.1997.tb00131.x

Wyllie, J., Carlson, J., \& Rosenberger, P. J. (2015). Does sexual-stimuli intensity and sexual self-schema influence female consumers' reactions toward sexualised advertising? An Australian perspective. Australasian Marketing Journal (AMJ), 23(3), 188-195.

doi:https://doi.org/10.1016/j.ausmj.2015.06.004 